\title{
Propagation of shear waves in an irregular magnetoelastic monoclinic layer sandwiched between two isotropic half-spaces
}

\author{
A. Chattopadhyay, S. Gupta, A.K. Singh and S.A. Sahu
}

\author{
Department of Applied Mathematics, Indian School of Mines (ISM),Dhanbad-826004, Jharkhand, India \\ E-mail: (amares.c@gmail.com (A. Chattopadhyay), "Corresponding author)
}

\begin{abstract}
In the present paper we study the propagation of horizontally polarised shear waves (SH waves) in an internal magnetoelastic monoclinic stratum with rectangular irregularity in lower interface and is sandwiched between two semi-infinite isotropic elastic media. The dispersion equation has been obtained. It is observed that the dispersion equation is in assertion with the classical Love-type wave equation, for isotropic layer sandwiched between two isotropic half spaces, in the absence of magnetic field and irregularity. The effects of depth of irregularity and monoclinic-magnetoelastic coupling parameters on dispersion curves are depicted by means of graphs. This study shows the remarkable effect of wave number, size of irregularity and magnetic field on the phase velocity.
\end{abstract}

Keywords: SH wave, magnetoelastic monoclinic, irregularity, dispersion equation, perturbation

\section{Introduction}

Many results of theoretical and experimental studies revealed that a real earth is considerably more complicated than the models presented earlier. This has led to a need for more realistic representation as a medium through which seismic waves propagate. The study of wave propagation in elastic medium with different boundaries is of great importance to seismologists as well as to geophysicists to understand and predict the seismic behaviour at the different margins of earth.

The propagation of Love waves has been studied by many authors with assuming different forms of irregularities at the interface. Bhattacharya (1962) discussed the dispersion curves for Love wave propagation in a transversely isotropic crustal layer with an irregularity in the interface. Chattopadhyay (1975) studied the Love wave propagation due to irregularity in the thickness of nonhomogeneous crustal layer. The wave propagation in crystalline media plays a very interesting role in geophysics and also in ultrasonic and signal processing. Chattopadhyay and Bandyopadhyay (1986) studied the propagation of shear waves in an infinite monoclinic crystal plate as well as in infinite anisotropic non-homogeneous monoclinic plate. The study of wave propagation in irregular boundaries is also of equal importance to seismologists and geophysicists. Ge and Chen (2007) proposed a direct boundary element method for calculating elastic wave propagation in two-dimensional irregularly stratified models. Further a more efficient approach was developed by same authors (Ge and Chen, 2008) for seismic-wave simulation in a stratified medium with irregular interfaces. In particular different authors have done the study of SH-wave propagation in different irregular mediums. Chattopadhyay et al. (1983) studied the propagation of SH guided wave in an internal stratum with parabolic irregularity in the lower interface. Campillo (2006) studied the case of a dome in a stratified medium for modelling of SH-wave propagation in an irregularly layered medium. Ding and Dravinski (1998) studied the scattering of SH-waves in irregular interfaces. Reflection and transmission of SH-wave at a corrugated interface has been studied before. Kaur et al. (2005) obtained the Reflection and transmission coefficients due to incident plane SH-waves at a corrugated interface between two isotropic, laterally and vertically heterogeneous visco-elastic solid half spaces. Tomar and Kaur (2007) studied the reflection and transmission of SH-waves at a corrugated interface between anisotropic elastic and viscoelastic solid half-spaces.

Keeping in the mind the characteristic and structure of earth, the medium of monoclinic is of core interest for seismologists to study the seismic wave propagation. Singh and Tomar (2007) studied the propagation of quasi-P wave at the interface between two monoclinic half-spaces. Kalyani et al. (2008) studied the propagation of SH waves in the plane of mirror symmetry of a 
monoclinic multilayered medium with displacement normal to the plane. Recently, the propagation of SH waves in an irregular monoclinic crustal layer has been studied by Chattopadhyay et al. (2008). In this paper we have discussed the propagation of SH waves in an irregular magnetoelastic monoclinic layer lying in between two isotropic half-spaces. The irregularity is in the form of rectangle. The perturbation technique indicated by Eringen and Samuels (1959) has been used. It is observed that phase velocity is strongly influenced by the depth of irregularity and magnetic field. These important peculiarities are highlighted by means of graphs.

\section{Formulation of the problem}



We consider the model, which is comprised of perfectly conducting magnetoelastic monoclinic irregular internal stratum, $M_{2}$ sandwiched between two isotropic elastic semi-infinite media $M_{1}$ (upper) and $M_{3}$ (lower) as shown in Figure 1 . The irregularity is considered in the form of rectangle on the lower interface of the internal stratum. We assume the rectangular irregularity with the length $s$ and depth $H^{\prime}$. Let us choose the rectangular coordinate system with the origin $\mathrm{O}$ at the middle point of the span, s, of the irregularity. We have taken y-axis vertically downwards and $\mathrm{z}$ axis along the regular interface between the lower semi-infinite medium and the monoclinic magnetoelastic layer. Let $H$ be the thickness of the internal stratum except for the irregular region and $\mathrm{S}$ be the position of the source on the y-axis at a depth $d$ below the origin $\left(d>H^{\prime}\right)$.

The equation of the interface between layer and lower half space is defined as

$$
y=\varepsilon h(z)
$$

where

$$
h(z)= \begin{cases}0 & \text { for } z \leq \frac{-s}{2}, z \geq \frac{s}{2} \\ f(z) & \text { for } \frac{-s}{2} \leq z \leq \frac{s}{2}\end{cases}
$$


where $\varepsilon=\frac{H^{\prime}}{s}$ and $\varepsilon<<1$.

Let us consider $\mu_{r}, \rho_{r}, u_{r}(r=1,2,3)$ as the rigidities, densities and displacements of the upper half space, sandwiched layer, lower half space respectively.

For upper $\left(\mathrm{M}_{1}\right)$ and lower $\left(\mathrm{M}_{3}\right)$ semi-infinite medium,

$$
\frac{\partial^{2} u_{1}}{\partial y^{2}}+\frac{\partial^{2} u_{1}}{\partial z^{2}}=\frac{1}{\beta_{1}^{2}} \frac{\partial^{2} u_{1}}{\partial t^{2}}
$$

and

$$
\frac{\partial^{2} u_{3}}{\partial y^{2}}+\frac{\partial^{2} u_{3}}{\partial z^{2}}=\frac{1}{\beta_{3}^{2}} \frac{\partial^{2} u_{3}}{\partial t^{2}}
$$

where $\beta_{1}=\sqrt{\frac{\mu_{1}}{\rho_{1}}}$ and $\beta_{3}=\sqrt{\frac{\mu_{3}}{\rho_{3}}}$ are the shear wave speed in the semi-infinite medium $\mathrm{M}_{1}$ and $\mathrm{M}_{3}$.

For the monoclinic magnetoelastic layer $\left(\mathrm{M}_{2}\right)$, the stain-displacement relations for monoclinic layer are

$$
S_{1}=\frac{\partial u}{\partial x}, S_{2}=\frac{\partial v}{\partial y}, S_{3}=\frac{\partial w}{\partial z}, S_{4}=\frac{\partial w}{\partial y}+\frac{\partial v}{\partial z}, S_{5}=\frac{\partial u}{\partial z}+\frac{\partial w}{\partial x}, S_{6}=\frac{\partial v}{\partial x}+\frac{\partial u}{\partial y}
$$

where $u, v, w$ are displacement components in the direction $\mathrm{x}, \mathrm{y}, \mathrm{z}$ respectively, and

$S_{i}(i=1,2, \ldots, 6)$ are the strain components.

Also, the stress-strain relation for a rotated $\mathrm{y}$-cut quartz plate, which exhibits monoclinic symmetry with $\mathrm{x}$ being the diagonal axis are

$$
\begin{aligned}
& T_{1}=C_{11} S_{1}+C_{12} S_{2}+C_{13} S_{3}+C_{14} S_{4}, \\
& T_{2}=C_{12} S_{1}+C_{22} S_{2}+C_{23} S_{3}+C_{24} S_{4}, \\
& T_{3}=C_{13} S_{1}+C_{23} S_{2}+C_{33} S_{3}+C_{34} S_{4}, \\
& T_{4}=C_{14} S_{1}+C_{24} S_{2}+C_{34} S_{3}+C_{44} S_{4}, \\
& T_{5}=C_{55} S_{5}+C_{56} S_{6}, \\
& T_{6}=C_{56} S_{5}+C_{66} S_{6}
\end{aligned}
$$

where $T_{i}(i=1,2, \ldots, 6)$ are the stress components and $C_{i j}=C_{j i}(i=1,2, \ldots, 6)$ are the elastic constants.

Equations governing the propagation of small elastic disturbances in a perfectly conducting monoclinic medium having electromagnetic force $\mathbf{J} \times \mathbf{B}$ (the Lorentz force, $\mathbf{J}$ being the electric current density and $\mathbf{B}$ being the magnetic induction vector) as the only body force are

$$
\begin{aligned}
& \frac{\partial T_{1}}{\partial x}+\frac{\partial T_{6}}{\partial y}+\frac{\partial T_{5}}{\partial z}+(J \times B)_{x}=\rho_{2} \frac{\partial^{2} u_{2}}{\partial t^{2}}, \\
& \frac{\partial T_{6}}{\partial x}+\frac{\partial T_{2}}{\partial y}+\frac{\partial T_{4}}{\partial z}+(J \times B)_{y}=\rho_{2} \frac{\partial^{2} v_{2}}{\partial t^{2}}, \\
& \frac{\partial T_{5}}{\partial x}+\frac{\partial T_{4}}{\partial y}+\frac{\partial T_{3}}{\partial z}+(J \times B)_{z}=\rho_{2} \frac{\partial^{2} w_{2}}{\partial t^{2}}
\end{aligned}
$$

where $\rho_{2}$ is the density of the layer.

For SH wave propagating in the $\mathrm{z}$ - direction and causing displacement in the $\mathrm{x}$ - direction only, we shall assume that

$$
u_{2}=u_{2}(y, z, t), v_{2}=w_{2}=0 \text { and } \frac{\partial}{\partial x} \equiv 0 .
$$

Using Eqs. (4) and (7), the stress-strain relation (5) becomes 


$$
\begin{aligned}
& T_{1}=T_{2}=T_{3}=T_{4}=0, \\
& T_{5}=C_{55} \frac{\partial u_{2}}{\partial z}+C_{56} \frac{\partial u_{2}}{\partial y}, \\
& T_{6}=C_{56} \frac{\partial u_{2}}{\partial z}+C_{66} \frac{\partial u_{2}}{\partial y} .
\end{aligned}
$$

Using Eq. (8) in Eq. (6), we have

$$
C_{66} \frac{\partial^{2} u_{2}}{\partial y^{2}}+2 C_{56} \frac{\partial^{2} u_{2}}{\partial y \partial z}+C_{55} \frac{\partial^{2} u_{2}}{\partial z^{2}}+(J \times B)_{x}=\rho_{2} \frac{\partial^{2} u_{2}}{\partial t^{2}} .
$$

The well known Maxwell's equations governing the electromagnetic field are $\nabla \cdot \boldsymbol{B}=0, \nabla \times \boldsymbol{E}=-\frac{\partial \boldsymbol{B}}{\partial t}, \nabla \times \boldsymbol{H}=\boldsymbol{J}$ with

$$
\boldsymbol{B}=\mu_{e} \boldsymbol{H}, \boldsymbol{J}=\sigma\left(\boldsymbol{E}+\frac{\partial u_{i}}{\partial t} \times \boldsymbol{B}\right)
$$

where $\boldsymbol{E}$ is the induced electric field, $\boldsymbol{J}$ is the current density vector and magnetic field $\boldsymbol{H}$ includes both primary and induced magnetic fields. $\mu_{e}$ and $\sigma$ are the induced permeability and conduction coefficient respectively.

The liberalized Maxwell's stress tensor $\left(\tau_{i j}^{0}\right)^{M_{x}}$ due to the magnetic field is given by $\left(\tau_{i j}^{0}\right)^{M_{x}}=\mu_{e}\left(H_{i} h_{j}+H_{j} h_{i}-H_{k} h_{k} \delta_{i j}\right)$.

Let $\boldsymbol{H}=\left(H_{1}, H_{2}, H_{3}\right)$ and $\boldsymbol{h}_{i}=\left(h_{1}, h_{2}, h_{3}\right)$ where $\boldsymbol{h}_{i}$ is the change in the magnetic field. In writing the above equations, we have neglected the displacement current.

From Eq. (10), we get

$$
\nabla^{2} \boldsymbol{H}=\mu_{e} \sigma\left\{\frac{\partial \boldsymbol{H}}{\partial t}+\nabla \times\left(\frac{\partial u_{i}}{\partial t} \times \boldsymbol{H}\right)\right\} .
$$

In component form, Eq. (11) can be written as

$$
\begin{aligned}
& \frac{\partial H_{1}}{\partial t}=\frac{1}{\mu_{e} \sigma} \nabla^{2} H_{1}+\frac{\partial\left(H_{2} \frac{\partial u_{2}}{\partial t}\right)}{\partial y}+\frac{\partial\left(H_{3} \frac{\partial u_{2}}{\partial t}\right)}{\partial z}, \\
& \frac{\partial H_{2}}{\partial t}=\frac{1}{\mu_{e} \sigma} \nabla^{2} H_{2}, \\
& \frac{\partial H_{3}}{\partial t}=\frac{1}{\mu_{e} \sigma} \nabla^{2} H_{3} .
\end{aligned}
$$

For perfectly conducting medium, $\sigma \rightarrow \infty$ the equation (12) become

$$
\frac{\partial H_{2}}{\partial t}=\frac{\partial H_{3}}{\partial t}=0
$$

and

$$
\frac{\partial H_{1}}{\partial t}=\frac{\partial\left(H_{2} \frac{\partial u_{2}}{\partial t}\right)}{\partial y}+\frac{\partial\left(H_{3} \frac{\partial u_{2}}{\partial t}\right)}{\partial z} .
$$

It is clear from Eq. (13) that there is no perturbation in $H_{2}$ and $H_{3}$, however from Eq. (14) there may be perturbation in $H_{1}$. Therefore, taking small perturbation, say $h_{1}$ in $H_{1}$, we have

$$
H_{1}=H_{01}+h_{1}, H_{2}=H_{02} \text { and } H_{3}=H_{03} \text {, where }\left(H_{01}, H_{02}, H_{03}\right) \text { are components of the initial magnetic field } \boldsymbol{H}_{0} \text {. }
$$

We can write $\boldsymbol{H}_{0}=\left(0, H_{0} \cos \phi, H_{0} \sin \phi\right)$, where $H_{0}=\left|\boldsymbol{H}_{0}\right|$ and $\phi$ is the angle at which the wave crosses the magnetic field. Thus we have

$$
\boldsymbol{H}=\left(h_{1}, H_{0} \cos \phi, H_{0} \sin \phi\right) .
$$

We shall take initial value of $h_{1}$ as $h_{1}=0$. Using Eq. (15) in Eq. (14), we get 


$$
\frac{\partial h_{1}}{\partial t}=\frac{\partial\left(H_{0} \cos \phi \frac{\partial u_{2}}{\partial t}\right)}{\partial y}+\frac{\partial\left(H_{0} \sin \phi \frac{\partial u_{2}}{\partial t}\right)}{\partial z} .
$$

Integrating with respect to $t$, we get

$$
h_{1}=H_{0} \cos \phi \frac{\partial u_{2}}{\partial y}+H_{0} \sin \phi \frac{\partial u_{2}}{\partial z} .
$$

Considering $\nabla\left(\frac{H^{2}}{2}\right)=-(\nabla \times \boldsymbol{H}) \times \boldsymbol{H}+(\boldsymbol{H} . \nabla) \boldsymbol{H}$ and Eqs (10), we get

$$
\boldsymbol{J} \times \boldsymbol{B}=\mu_{e}\left\{-\nabla\left(\frac{H^{2}}{2}\right)+(\boldsymbol{H} . \nabla) \boldsymbol{H}\right\} .
$$

In the component form Eq. (18) can be written as

$$
(\boldsymbol{J} \times \boldsymbol{B})_{y}=(\boldsymbol{J} \times \boldsymbol{B})_{z}=0
$$

and

$$
(\boldsymbol{J} \times \boldsymbol{B})_{x}=\mu_{e} H_{0}{ }^{2}\left(\cos ^{2} \phi \frac{\partial^{2} u_{2}}{\partial y^{2}}+\sin 2 \phi \frac{\partial^{2} u_{2}}{\partial y \partial z}+\sin ^{2} \phi \frac{\partial^{2} u_{2}}{\partial z^{2}}\right) .
$$

Using Eqs. (9) and (19), we find the equation of motion for the magnetoelastic monoclinic layer in the form

$$
M_{66} \frac{\partial^{2} u_{2}}{\partial y^{2}}+2 M_{56} \frac{\partial^{2} u_{2}}{\partial y \partial z}+M_{55} \frac{\partial^{2} u_{2}}{\partial z^{2}}=\rho_{2} \frac{\partial^{2} u_{2}}{\partial t^{2}} .
$$

where

$$
\begin{aligned}
& M_{66}=C_{66}\left(1+m_{H} \cos ^{2} \phi\right), \\
& M_{55}=C_{66}\left(\frac{C_{55}}{C_{66}}+m_{H} \sin ^{2} \phi\right), \\
& M_{56}=C_{66}\left(\frac{C_{56}}{C_{66}}+m_{H} \cos \phi \sin \phi\right) .
\end{aligned}
$$

Where $m_{H}=\frac{\mu_{e} H_{0}^{2}}{C_{66}}$ is monoclinic-magnetoelastic coupling parameter. Here in equations (2), (3) and (20) suffixes " 1 ”, "2" and “3” are used to represent the upper semi-infinite medium $\left(M_{1}\right)$, monoclinic layer $\left(M_{2}\right)$ and lower semi-infinite medium $\left(M_{3}\right)$ respectively.

The boundary conditions are as follows:

(i) The stresses are continuous at the interface of $M_{1}$ and $M_{2}$,

$$
\mu_{1} \frac{\partial u_{1}}{\partial y}=M_{56} \frac{\partial u_{2}}{\partial z}+M_{66} \frac{\partial u_{2}}{\partial y} \quad \text { at } y=-H .
$$

(ii) The Displacements are continuous at the interface of $\mathrm{M}_{1}$ and $\mathrm{M}_{2}$,

$$
u_{1}=u_{2} \text { at } y=-H \text {. }
$$

(iii) The stresses are continuous at the interface of $M_{2}$ and $M_{3}$,

$$
\left(M_{56}-M_{55} \varepsilon h^{\prime}\right) \frac{\partial u_{2}}{\partial z}+\left(M_{66}-M_{56} \varepsilon h^{\prime}\right) \frac{\partial u_{2}}{\partial y}=\mu_{3}\left(\frac{\partial u_{3}}{\partial y}-\varepsilon h^{\prime} \frac{\partial u_{3}}{\partial z}\right) \quad \text { at } y=\varepsilon h(z)
$$

where $h^{\prime}=\frac{d h(z)}{d z}$.

(iv) The Displacements are continuous at the interface of $M_{2}$ and $M_{3}$,

$$
u_{2}=u_{3} \text { at } y=\operatorname{ch}(z) \text {. }
$$




\section{Solution of the problem}

Let us assume $u_{i}=U_{i}(y, z) e^{\text {iot }}(i=1,2,3)$. Under this assumption equations (2), (3) and (20) reduce to

$$
\begin{aligned}
& \frac{\partial^{2} U_{1}}{\partial y^{2}}+\frac{\partial^{2} U_{1}}{\partial z^{2}}+\frac{\omega^{2}}{\beta_{1}^{2}} U_{1}=0, \\
& \frac{\partial^{2} U_{3}}{\partial y^{2}}+\frac{\partial^{2} U_{3}}{\partial z^{2}}+\frac{\omega^{2}}{\beta_{3}^{2}} U_{3}=0
\end{aligned}
$$

and

$$
M_{66} \frac{\partial^{2} U_{2}}{\partial y^{2}}+2 M_{56} \frac{\partial^{2} U_{2}}{\partial y \partial z}+M_{55} \frac{\partial^{2} U_{2}}{\partial z^{2}}+\rho_{2} \omega^{2} U_{2}=0 .
$$

where $\omega$ is the circular frequency.

Defining the Fourier transform $\bar{U}_{r}(y, \eta)$ of $U_{r}(y, z)(r=1,2,3)$ as

$$
\bar{U}_{r}(y, \eta)=\int_{-\infty}^{\infty} U_{r}(y, z) e^{i \eta z} d z
$$

We find the inverse Fourier transform as

$$
U_{r}(y, z)=\frac{1}{2 \pi} \int_{-\infty}^{\infty} \bar{U}_{r}(y, \eta) e^{-i \eta z} d \eta .
$$

Using Fourier transform in equations (26), (27) and (28), we have

$$
\begin{aligned}
& \frac{d^{2} \bar{U}_{1}}{d y^{2}}-p_{1}{ }^{2} \bar{U}_{1}=0, \\
& \frac{d^{2} \bar{U}_{3}}{d y^{2}}-p_{3}{ }^{2} \bar{U}_{3}=0
\end{aligned}
$$

and

$$
\frac{d^{2} \bar{U}_{2}}{d y^{2}}+a \frac{d \bar{U}_{2}}{d y}+p_{2}^{2} \bar{U}_{2}=0
$$

where

$$
p_{1}^{2}=\eta^{2}-\frac{\omega^{2}}{\beta_{1}^{2}}, p_{2}{ }^{2}=\frac{\omega^{2}}{\beta_{2}^{2}}-\frac{M_{55}}{M_{66}} \eta^{2}, p_{3}{ }^{2}=\eta^{2}-\frac{\omega^{2}}{\beta_{3}^{2}} \text { and } a=-2 i \eta \frac{M_{56}}{M_{66}} \text {. }
$$

The appropriate solutions of Eqs. (29) and (30) can be taken as

$$
\bar{U}_{1}=A e^{p_{1} y}
$$

and

$$
\bar{U}_{3}=E e^{-p_{3} y} .
$$

The solution of Eq. (31) is

where

$$
\bar{U}_{2}=e^{-\frac{a}{2} y}(B \cos p y+D \sin p y)
$$

$$
p=\sqrt{p_{2}{ }^{2}-\frac{a^{2}}{4}}=\left(\frac{\omega^{2}}{\beta_{2}{ }^{2}}-\frac{M_{55}}{M_{66}} \eta^{2}+\frac{M_{56}{ }^{2}}{M_{66}{ }^{2}} \eta^{2}\right)^{1 / 2} .
$$

The constants $A, B, D$ and $E$ are functions of $\eta$.

The displacements in $\mathrm{M}_{1}, \mathrm{M}_{2}$ and $\mathrm{M}_{3}$ are

$$
U_{1}(y, z)=\frac{1}{2 \pi} \int_{-\infty}^{\infty} A e^{p_{1} y} e^{-i \eta \eta z} d \eta,
$$




$$
U_{2}(y, z)=\frac{1}{2 \pi} \int_{-\infty}^{\infty} e^{-\frac{a}{2} y}(B \cos p y+D \sin p y) e^{-i \eta \eta z} d \eta
$$

and

$$
U_{3}(y, z)=\frac{1}{2 \pi} \int_{-\infty}^{\infty}\left(E e^{-p_{3} y}+\frac{2}{p_{3}} e^{p_{3} y} e^{-p_{3} d}\right) e^{-i \eta z} d \eta .
$$

where the second term in the integrand of $U_{3}$ is introduced due to the effect of source at $S$ in $M_{3}$ (Willis, 1948). We set the following approximations due to small value of $\varepsilon$

$$
B \cong B_{0}+B_{1} \varepsilon, D \cong D_{0}+D_{1} \varepsilon, E \cong E_{0}+E_{1} \varepsilon .
$$

Since the boundary is not uniform at the interface of $\mathrm{M}_{2}$ and $\mathrm{M}_{3}$ so the term $B, D$ and $E$ appearing in Eq.(38) are also function of $\varepsilon$, expanding these terms in ascending powers of $\varepsilon$ and keeping in view that $\varepsilon$ is small and so retaining the term up to the first order of $\varepsilon$, hence approximated as in Eq.(38). These assumptions are justified in the real earth model where the depth $H^{\prime}$ of the irregular boundary is too small with respect to the length of the boundary $s$.

Also for small $\varepsilon$, following approximations can be accepted

$$
e^{ \pm v \varepsilon h} \cong 1 \pm v \varepsilon h, \cos p \varepsilon h \cong 1, \sin p \varepsilon h \cong p \varepsilon h .
$$

where $v$ is any quantity.

Using the boundary conditions (i), (ii) and (iv), we have the following equations

$$
\begin{aligned}
& M_{56}\left\{\left(B_{0}+B_{1} \varepsilon\right) \cos p H-\left(D_{0}+D_{1} \varepsilon\right) \sin p H\right\}(-i \eta) e^{\frac{a H}{2}}-M_{66}\left\{\left(B_{0}+B_{1} \varepsilon\right) \cos p H\right. \\
& \left.-\left(D_{0}+D_{1} \varepsilon\right) \sin p H\right\}\left(\frac{a}{2}\right) e^{\frac{a H}{2}}+M_{66}\left\{\left(B_{0}+B_{1} \varepsilon\right) \sin p H+\left(D_{0}+D_{1} \varepsilon\right) \cos p H\right\}(p) e^{\frac{a H}{2}} \\
& =\mu_{1} A p_{1} e^{-p_{1} H}, \\
& \left\{\left(B_{0}+B_{1} \varepsilon\right) \cos p H-\left(D_{0}+D_{1} \varepsilon\right) \sin p H\right\} e^{\frac{a H}{2}}=A e^{-p_{1} H}
\end{aligned}
$$

and

$$
\begin{aligned}
& \varepsilon \int_{-\infty}^{\infty}\left(\frac{a}{2} B_{0}-p D_{0}-p_{3} E_{0}+2 e^{-p_{3} d}\right) h(z) e^{-i \eta z} d \eta \\
& =\int_{-\infty}^{\infty}\left\{\left(B_{0}-E_{0}-\frac{2}{p_{3}} e^{-p_{3} d}\right)+\varepsilon\left(B_{1}-E_{1}\right)\right\} e^{-i \eta z} d \eta .
\end{aligned}
$$

Now applying the Fourier transform of $h(z)$ defined as

$$
\bar{h}(\lambda)=\int_{-\infty}^{\infty} h(z) e^{i \lambda z} d z
$$

so that

$$
h(z)=\frac{1}{2 \pi} \int_{-\infty}^{\infty} \bar{h}(\lambda) e^{-i \lambda z} d \lambda
$$

and

$$
h^{\prime}(z)=-\frac{i}{2 \pi} \int_{-\infty}^{\infty} \lambda \bar{h}(\lambda) e^{-i \lambda z} d \lambda .
$$

Now applying the transform (43) and (44) on (41), we have

$$
\begin{aligned}
& \frac{\varepsilon}{2 \pi} \int_{-\infty}^{\infty}\left[\int_{-\infty}^{\infty}\left(\frac{a}{2} B_{0}-p D_{0}-p_{3} E_{0}+2 e^{-p_{3} d}\right) \bar{h}(\lambda) e^{-i(\eta+\lambda) z} d \eta\right] d \lambda \\
& =\int_{-\infty}^{\infty}\left\{\left(B_{0}-E_{0}-\frac{2}{p_{3}} e^{-p_{3} d}\right)+\varepsilon\left(B_{1}-E_{1}\right)\right\} e^{-i \eta \eta z} d \eta .
\end{aligned}
$$


Taking $\eta+\lambda=k$ for the inner integral in the left-hand side of the Eq. (45), so that $\lambda$ may be treated as a constant such that $d \eta=d k$, replacing $\eta$ by $k$ in the right-hand side of the Eq.(45), and finally after taking Fourier transform as defined above we can have

$$
\left(B_{0}-E_{0}-\frac{2}{p_{3}} e^{-p_{3} d}\right)+\varepsilon\left(B_{1}-E_{1}\right)=\varepsilon R_{1}(k)
$$

where

$$
R_{1}(k)=\frac{1}{2 \pi} \int_{-\infty}^{\infty}\left[\frac{a}{2} B_{0}-p D_{0}-p_{3} E_{0}+2 e^{-p_{3} d}\right]^{\eta=k-\lambda} \bar{h}(\lambda) d \lambda
$$

From the boundary condition (iii), we obtain

$$
\begin{aligned}
& M_{66}\left\{\frac{a}{2} B_{0}-p D_{0}+\varepsilon\left(\frac{a}{2} B_{1}-p D_{1}\right)\right\}+i M_{56} k\left(B_{0}+B_{1} \varepsilon\right)-\mu_{3}\left(p_{3} E_{0}-2 e^{-p_{3} d}+\varepsilon p_{3} E_{1}\right) \\
& =\varepsilon R_{2}(k)
\end{aligned}
$$

where

$$
\begin{aligned}
R_{2}(k)= & \frac{1}{2 \pi} \int_{-\infty}^{\infty}\left[\left\{M_{66}\left(\frac{a^{2}}{4} B_{0}-p^{2} B_{0}-a p D_{0}\right)+M_{56}\left(\frac{a}{2} B_{0}-p D_{0}\right) i k-\mu_{3}\left(p_{3}{ }^{2} E_{0}+2 p_{3} e^{-p_{3} d}\right)\right\}\right. \\
& \left.+\lambda\left\{i M_{56}\left(D_{0} p-\frac{a}{2} B_{0}\right)+M_{55} k B_{0}-k \mu_{3}\left(E_{0}+\frac{2}{p_{3}} e^{-p_{3} d}\right)\right\}\right]^{\eta=k-\lambda} \bar{h}(\lambda) d \lambda .
\end{aligned}
$$

Equating the absolute term ( term not containing $\varepsilon$ ) and the coefficient of $\varepsilon$ from Eqs. (39),

(40), (46) and (48) we get

$$
\begin{aligned}
& B_{0}\left(-i \eta M_{56} \cos p H-\frac{a}{2} M_{66} \cos p H+p M_{66} \sin p H\right) e^{\frac{a H}{2}} \\
& +D_{0}\left(i \eta M_{56} \sin p H+\frac{a}{2} M_{66} \sin p H+p M_{66} \cos p H\right) e^{\frac{a H}{2}}=A p_{1} \mu_{1} e^{-p_{1} H}, \\
& B_{1}\left(-i \eta M_{56} \cos p H-\frac{a}{2} M_{66} \cos p H+p M_{66} \sin p H\right) e^{\frac{a H}{2}} \\
& +D_{1}\left(i \eta M_{56} \sin p H+\frac{a}{2} M_{66} \sin p H+p M_{66} \cos p H\right) e^{\frac{a H}{2}}=0, \\
& \left(B_{0} \cos p H-D_{0} \sin p H\right) e^{\frac{a H}{2}}=A e^{-p_{1} H}, \\
& \left(B_{1} \cos p H-D_{1} \sin p H\right) e^{\frac{a H}{2}}=0, \\
& B_{0}-E_{0}-\frac{2}{p_{3}} e^{-p_{3} d}=0, \\
& B_{1}-E_{1}=R_{1}(k), \\
& B_{0}\left(\frac{a}{2} M_{66}+i k M_{56}\right)-\left(p M_{66}\right) D_{0}-\mu_{3}\left(p_{3} E_{0}-2 e^{-p_{3} d}\right)=0,
\end{aligned}
$$

and

$$
B_{1}\left(\frac{a}{2} M_{66}+i k M_{56}\right)-\left(p M_{66}\right) D_{1}-\left(\mu_{3} p_{3}\right) E_{1}=R_{2}(k) .
$$

Solving the above eight equations, we get 


$$
\begin{aligned}
& B_{0}=\frac{-8 \mu_{3} e^{-p_{3} d}}{G(k)}\left(2 i k M_{56} \tan p H+a M_{66} \tan p H+2 p M_{66}+2 p_{1} \mu_{1} \tan p H\right) \text {, } \\
& D_{0}=\frac{-8 \mu_{3} e^{-p_{3} d}}{G(k)}\left(2 i k M_{56}+a M_{66}-2 p M_{66} \tan p H+2 p_{1} \mu_{1}\right) \text {, } \\
& E_{0}=\frac{-2 e^{-p_{3} d}}{p_{3} G(k)}\left(E_{2}+E_{3}\right) \text {, } \\
& B_{1}=\frac{-2\left(\mu_{3} p_{3} R_{1}-R_{2}\right)}{G(k)}\left(2 i k M_{56} \tan p H+a M_{66} \tan p H+2 p M_{66}+2 p_{1} \mu_{1} \tan p H\right) \text {, } \\
& D_{1}=\frac{-2\left(\mu_{3} p_{3} R_{1}-R_{2}\right)}{G(k)}\left(2 i k M_{56}+a M_{66}-2 p M_{66} \tan p H+2 p_{1} \mu_{1}\right) \text {, } \\
& E_{1}=\frac{\left(E_{4}+E_{5}\right)}{G(k)} \text {, } \\
& A=\left(B_{0} \cos p H-D_{0} \sin p H\right) e^{\left(\frac{a}{2}+p_{1}\right) H}, \\
& E_{2}=4 i k p_{3} \mu_{3} M_{56} \tan p H+2 a p_{3} \mu_{3} M_{66} \tan p H+4 p_{3} \mu_{3} p M_{66}+4 p_{3} \mu_{3} p_{1} \mu_{1} \tan p H \text {, } \\
& E_{3}=4 p^{2} M_{66}{ }^{2} \tan p H-4 p p_{1} \mu_{1} M_{66}+4 i k a M_{56} M_{66} \tan p H+a^{2} M_{66}{ }^{2} \tan p H \\
& +2 a p_{1} \mu_{1} M_{66} \tan p H-4 k^{2} M_{56}{ }^{2} \tan p H+4 i k p_{1} \mu_{1} M_{56} \tan p H, \\
& E_{4}=4 i k R_{2} M_{56} \tan p H+2 a R_{2} M_{66} \tan p H+4 p R_{2} M_{66}+4 p_{1} \mu_{1} R_{2} \tan p H-4 i k a R_{1} M_{56} M_{66} \tan p H \\
& -4 i k R_{1} p_{1} \mu_{1} M_{56} \tan p H, \\
& E_{5}=-4 p^{2} R_{1} M_{66}{ }^{2} \tan p H+4 p p_{1} \mu_{1} R_{1} M_{66}-a^{2} R_{1} M_{66}{ }^{2} \tan p H-2 a p_{1} \mu_{1} R_{1} M_{66} \tan p H+4 k^{2} R_{1} M_{56}{ }^{2} \tan p H \text {, } \\
& G(k)=4 p^{2} M_{66}{ }^{2} \tan p H-4 p p_{1} \mu_{1} M_{66}+4 i k a M_{56} M_{66} \tan p H+a^{2} M_{66}{ }^{2} \tan p H+2 a p_{1} \mu_{1} M_{66} \tan p H \\
& -4 k^{2} M_{56}{ }^{2} \tan p H+4 i k p_{1} \mu_{1} M_{56} \tan p H-4 i k p_{3} \mu_{3} M_{56} \tan p H-2 p_{3} \mu_{3} a M_{66} \tan p H \\
& -4 p_{3} \mu_{3} p M_{66}-4 p_{3} \mu_{3} p_{1} \mu_{1} \tan p H \text {. }
\end{aligned}
$$

With the help of above obtained values the displacement in the monoclinic layer is given by

$$
\begin{aligned}
U_{2}=- & \frac{1}{2} \int_{-\infty}^{\infty} \frac{8 \mu_{3} e^{-p_{3} d} e^{-\frac{a}{2} y}}{G(k)}\left[1+\frac{\varepsilon\left(\mu_{3} p_{3} R_{1}-R_{2}\right)}{4 \mu_{3} e^{-p_{3} d}}\right] \\
& \times\left\{\left(2 i k M_{56} \tan p H+a M_{66} \tan p H+2 p M_{66}+2 p_{1} \mu_{1} \tan p H\right) \cos p y\right. \\
& \left.+\left(2 i k M_{56}+a M_{66}-2 p M_{66} \tan p H+2 p_{1} \mu_{1}\right) \sin p y\right\} e^{-i k z} d k .
\end{aligned}
$$

From Eq. (1), the interface for the rectangular irregularity gives

$$
\bar{h}(\lambda)=\frac{2 s}{\lambda} \sin \left(\frac{\lambda s}{2}\right) \text {. }
$$

Further on simplification, we get

$$
\mu_{3} p_{3} R_{1}-R_{2}=\frac{2 s \mu_{3}}{\pi} \int_{-\infty}^{\infty}\{\psi(k-\lambda)+\psi(k+\lambda)\} \frac{1}{\lambda} \sin \left(\frac{\lambda s}{2}\right) d \lambda,
$$

where

and

$$
\psi(k-\lambda)=\left[\left(B_{2}+B_{3}+B_{4}+B_{5}+B_{6}+B_{7}+B_{8}+B_{9}+B_{10}+B_{11}+B_{12}\right) \frac{e^{-p_{3} d}}{p_{3} G(k)}\right]^{\eta=k-\lambda}
$$




$$
\begin{aligned}
& B_{2}=-2 a^{2} p_{3}{ }^{2} \mu_{3} M_{66} \tan p H-2 a^{2} p p_{3} M_{66}{ }^{2}-8 p^{3} p_{3} M_{66}{ }^{2}-8 p^{2} p_{1} p_{3} \mu_{1} M_{66} \tan p H+2 p_{3}{ }^{2} E(k) \text {, } \\
& B_{3}=-4 i a k \mu_{3} p_{3}{ }^{2} M_{66} \tan p H-8 p^{2} p_{3}{ }^{2} \mu_{3} M_{66} \tan p H+4 a p^{2} p_{3} M_{66}{ }^{2} \tan p H \text {, } \\
& B_{4}=8 i k p p_{3}{ }^{2} \mu_{3} M_{66}+4 i k a^{2} p_{3} M_{56} M_{66} \tan p H+a^{3} p_{3} M_{66}{ }^{2} \tan p H-8 i a k p p_{3} M_{56} M_{66} \text {, } \\
& B_{5}=-4 a p_{1} p_{3}{ }^{2} \mu_{1} \mu_{3} \tan p H+8 p p_{1} p_{3}{ }^{2} \mu_{1} \mu_{3}+2 a^{2} p_{1} p_{3} \mu_{1} M_{66} \tan p H-8 a p p_{1} p_{3} \mu_{1} M_{66} \text {, } \\
& B_{6}=-8 \lambda k p p_{3} M_{56}{ }^{2}+4 \lambda a k p_{3} M_{56}{ }^{2} \tan p H+8 i \lambda k^{2} p_{3} M_{55} M_{56} \tan p H+8 \lambda k p p_{3} M_{55} M_{66} \text {, } \\
& B_{7}=-4 i \lambda k^{2} p_{3} \mu_{3} M_{56} \tan p H-2 a \lambda k \mu_{3} p_{3} M_{66} \tan p H+4 a \lambda k p_{3} M_{55} M_{66} \tan p H \text {, } \\
& B_{8}=-4 \lambda k p^{2} M_{66}{ }^{2} \tan p H-4 i a \lambda k^{2} M_{56} M_{66} \tan p H-4 \lambda k p p_{3} \mu_{3} M_{66}+\lambda k E(k) \text {, } \\
& B_{9}=4 \lambda k^{3} M_{56}{ }^{2} \tan p H-a^{2} \lambda k M_{66}{ }^{2} \tan p H-4 a k^{2} p_{3} M_{56}{ }^{2} \tan p H-4 \lambda k p p_{1} \mu_{1} M_{66} \text {, } \\
& B_{10}=-8 i \lambda p^{2} p_{3} M_{56} M_{66} \tan p H-2 i a^{2} \lambda p_{3} M_{56} M_{66} \tan p H+8 k^{2} p p_{3} M_{56}{ }^{2} \text {, } \\
& B_{11}=8 i \lambda p p_{1} p_{3} \mu_{1} M_{56}-4 i a \lambda p_{1} p_{3} \mu_{1} M_{56} \tan p H+8 \lambda k p_{1} p_{3} \mu_{1} M_{55} \tan p H \text {, } \\
& B_{12}=2 a \lambda k p_{1} \mu_{1} M_{66} \tan p H+4 i \lambda k^{2} p_{1} \mu_{1} M_{56} \tan p H-4 \lambda k p_{1} p_{3} \mu_{1} \mu_{3} \tan p H \text {. }
\end{aligned}
$$

Here the argument of $\psi(k-\lambda)$ is because of $\eta+\lambda=k$. Following the asymptotic formula of Willis (1948) and neglecting the terms containing $2 / s$ and higher powers of $2 / s$ for large $s$, we get

$$
\int_{-\infty}^{\infty}\{\psi(k-\lambda)+\psi(k+\lambda)\} \frac{1}{\lambda} \sin \left(\frac{\lambda s}{2}\right) d \lambda \cong \frac{\pi}{2} 2 \psi(k)=\pi \psi(k) \text {, (cf. Tranter, 1966). }
$$

Hence on using Eq. (61) on Eq. (60) one can have

$$
\mu_{3} p_{3} R_{1}-R_{2}=2 s \mu_{3} \psi(k)=2 \mu_{3} \frac{H^{\prime}}{\varepsilon} \psi(k) .
$$

Therefore, in view of Eq. (62) the displacement in the magnetoelastic monoclinic layer is

$$
\begin{aligned}
U_{2}=-\frac{1}{2 \pi} \int_{-\infty}^{\infty} \frac{8 \mu_{3} e^{-p_{3} d} e^{-\frac{a}{2} y}}{G(k)\left[1-\frac{H^{\prime}}{2} \psi(k) e^{p_{3} d}\right]}\left\{\left(2 i k M_{56} \tan p H+a M_{66} \tan p H+2 p M_{66}\right.\right. \\
\left.\left.\quad+2 p_{1} \mu_{1} \tan p H\right) \cos p y+\left(2 i k M_{56}+a M_{66}-2 p M_{66} \tan p H+2 p_{1} \mu_{1}\right) \sin p y\right\} e^{-i k z} d k .
\end{aligned}
$$

Since the value of this integral depends entirely on the contribution of the poles of the Integrand, the dispersion equation for SH waves is given by

$$
G(k)\left[1-\frac{H^{\prime}}{2} \psi(k) e^{p_{3} d}\right]=0 .
$$

The poles are located at the roots of above equation which has been examined in our study of shear waves (cf. Achenbach, 1975). Eq. (63) may be rewritten as

$$
\begin{aligned}
& D_{2}+D_{3}+D_{4}-\frac{H^{\prime}}{2}\left(D_{5}+D_{6}+D_{7}+D_{8}\right)=0 \\
& D_{2}=4 p^{2} M_{66}{ }^{2} \tan p H-4 p p_{1} \mu_{1} M_{66}+4 i k a M_{56} M_{66} \tan p H+a^{2} M_{66}{ }^{2} \tan p H, \\
& D_{3}=2 a p_{1} \mu_{1} M_{66} \tan p H-4 k^{2} M_{56}{ }^{2} \tan p H+4 i k p_{1} \mu_{1} M_{56} \tan p H-4 i k p_{3} \mu_{3} M_{56} \tan p H, \\
& D_{4}=-2 p_{3} \mu_{3} a M_{66} \tan p H-4 p_{3} \mu_{3} p M_{66}-4 p_{3} \mu_{3} p_{1} \mu_{1} \tan p H, \\
& D_{5}=-2 a^{2} p_{3}{ }^{2} \mu_{3} M_{66} \tan p H-2 a^{2} p_{3} M_{66}{ }^{2}-8 p^{2} p_{1} p_{3} \mu_{1} M_{66} \tan p H+2 p_{3}{ }^{2} E(k), \\
& D_{6}=-4 i a k \mu_{3} p_{3}{ }^{2} M_{66} \tan p H-8 p^{2} p_{3}{ }^{2} \mu_{3} M_{66} \tan p H+4 a p^{2} p_{3} M_{66}{ }^{2} \tan p H, \\
& D_{7}=8 i k p p_{3}{ }^{2} \mu_{3} M_{66}+4 i k a^{2} p_{3} M_{56} M_{66} \tan p H+a^{3} p_{3} M_{66}{ }^{2} \tan p H, \\
& D_{8}=-4 a k^{2} p_{3} M_{56}{ }^{2} \tan p H+8 k^{2} p p_{3} M_{56}{ }^{2}-8 i a k p p_{3} M_{56} M_{66}-8 p^{3} p_{3} M_{66}{ }^{2} .
\end{aligned}
$$


Let us assume that the wave is propagating along the surface with $C$ as the common wave velocity, and then we can set the following

$$
p=k P, p_{1}=k P_{1}, p_{3}=k P_{3} \text { and } a=k A
$$

where $P=\left(\frac{c^{2}}{\beta_{2}^{2}}-\frac{M_{55}}{M_{66}}+\frac{M_{56}{ }^{2}}{M_{66}{ }^{2}}\right)^{1 / 2}, P_{1}=\left(1-\frac{c^{2}}{\beta_{1}^{2}}\right)^{1 / 2}, P_{3}=\left(1-\frac{c^{2}}{\beta_{3}^{2}}\right)^{1 / 2}, A=-2 i \frac{M_{56}}{M_{66}}$.

In the view of above setting, Eq. (64) gives

$$
\tan \left\{\sqrt{\left(\frac{c^{2}}{\beta_{2}^{2}}-\frac{M_{55}}{M_{66}}+\frac{M_{56}^{2}}{M_{66}^{2}}\right)} k H\right\}=\frac{J_{1}}{L_{1}}+i \frac{J_{2}}{L_{2}}
$$

where

$$
\begin{aligned}
J_{1}= & \left(8 P P_{1} \mu_{1} M_{66}+8 P P_{3} \mu_{3} M_{66}+8 H^{\prime} k P P_{1} P_{3} \mu_{1} \mu_{3}-8 H^{\prime} k P_{1} P_{3} \mu_{1} M_{66}-8 H^{\prime} k P^{3} M_{66}{ }^{2}-8 H^{\prime} k P P_{3}{ }^{2} \mu_{3} M_{66}\right) \\
& \times\left(8 P^{2} M_{66}{ }^{2}-8 P_{1} P_{3} \mu_{1} \mu_{3}+8 H^{\prime} k P^{2} P_{3} \mu_{3} M_{66}-8 H^{\prime} k P^{2} P_{3} M_{66}{ }^{2}+8 H^{\prime} k P^{2} P_{1} \mu_{1} M_{66}+8 H^{\prime} k P_{3}^{2} P_{1} \mu_{1} \mu_{3}\right) \\
& +\left(8 H^{\prime} k P P_{3} \mu_{3} M_{56}+8 H^{\prime} k P P_{1} \mu_{1} M_{56}\right)\left(8 H^{\prime} k P^{2} M_{56} M_{66}-8 H^{\prime} k P_{1} P_{3} \mu_{1} \mu_{3} M_{56} / M_{66}\right), \\
L_{1}= & \left(8 P^{2} M_{66}{ }^{2}-8 P_{1} P_{3} \mu_{1} \mu_{3}+8 H^{\prime} k P^{2} P_{3} \mu_{3} M_{66}-8 H^{\prime} k P^{2} P_{3} M_{66}{ }^{2}+8 H^{\prime} k P^{2} P_{1} \mu_{1} M_{66}+8 H^{\prime} k P_{3}^{2} P_{1} \mu_{1} \mu_{3}\right)^{2} \\
+ & \left(8 H^{\prime} k P^{2} M_{56} M_{66}-8 H^{\prime} k P_{1} P_{3} \mu_{1} \mu_{3} M_{56} / M_{66}\right)^{2}, \\
J_{2}= & \left(8 H^{\prime} k P P_{3} \mu_{3} M_{56}+8 H^{\prime} k P P_{1} \mu_{1} M_{56}\right)\left(8 P^{2} M_{66}{ }^{2}-8 P_{1} P_{3} \mu_{1} \mu_{3}+8 H^{\prime} k P^{2} P_{3} \mu_{3} M_{66}-8 H^{\prime} k P^{2} P_{3} M_{66}{ }^{2}\right. \\
& \left.+8 H^{\prime} k P^{2} P_{1} \mu_{1} M_{66}+8 H^{\prime} k P_{3}^{2} P_{1} \mu_{1} \mu_{3}\right)-\left(8 H^{\prime} k P^{2} M_{56} M_{66}-8 H^{\prime} k P_{1} P_{3} \mu_{1} \mu_{3} M_{56} / M_{66}\right) \\
& \times\left(8 P P_{1} \mu_{1} M_{66}+8 P P_{3} \mu_{3} M_{66}+8 H^{\prime} k P P_{1} P_{3} \mu_{1} \mu_{3}-8 H^{\prime} k P P_{1} P_{3} \mu_{1} M_{66}-8 H^{\prime} k P^{3} M_{66}{ }^{2}-8 H^{\prime} k P P_{3}^{2} \mu_{3} M_{66}\right), \\
L_{2}= & \left(8 P^{2} M_{66}{ }^{2}-8 P_{1} P_{3} \mu_{1} \mu_{3}+8 H^{\prime} k P^{2} P_{3} \mu_{3} M_{66}-8 H^{\prime} k P^{2} P_{3} M_{66}{ }^{2}+8 H^{\prime} k P^{2} P_{1} \mu_{1} M_{66}+8 H^{\prime} k P_{3}^{2} P_{1} \mu_{1} \mu_{3}\right)^{2} \\
+ & \left(8 H^{\prime} k P^{2} M_{56} M_{66}-8 H^{\prime} k P_{1} P_{3} \mu_{1} \mu_{3} M_{56} / M_{66}\right)^{2} .
\end{aligned}
$$

The real part of the Eq. (65) gives the dispersion equation of SH waves. The dispersion relation for the SH waves is

$$
\tan \left\{\sqrt{\left(\frac{c^{2}}{\beta_{2}^{2}}-\frac{M_{55}}{M_{66}}+\frac{M_{56}{ }^{2}}{M_{66}{ }^{2}}\right)} k H\right\}=\frac{J_{1}}{L_{1}} .
$$

\section{Particular cases}

\section{Case 1:}

When $m_{H}=0$ the dispersion relation (66) reduces to

$$
\tan \left\{\sqrt{\left(\frac{c^{2}}{\beta_{2}^{2}}-\frac{C_{55}}{C_{66}}+\frac{C_{56}^{2}}{C_{66}^{2}}\right)} k H\right\}=\frac{J_{3}}{L_{3}}
$$

where

$$
\begin{aligned}
J_{3}= & \left(8 P P_{1} \mu_{1} C_{66}+8 P P_{3} \mu_{3} C_{66}+8 H^{\prime} k P P_{1} P_{3} \mu_{1} \mu_{3}-8 H^{\prime} k P P_{1} P_{3} \mu_{1} C_{66}-8 H^{\prime} k P^{3} C_{66}{ }^{2}-8 H^{\prime} k P P_{3}^{2} \mu_{3} C_{66}\right) \\
& \times\left(8 P^{2} C_{66}{ }^{2}-8 P_{1} P_{3} \mu_{1} \mu_{3}+8 H^{\prime} k P^{2} P_{3} \mu_{3} C_{66}-8 H^{\prime} k P^{2} P_{3} C_{66}{ }^{2}+8 H^{\prime} k P^{2} P_{1} \mu_{1} C_{66}+8 H^{\prime} k P_{3}^{2} P_{1} \mu_{1} \mu_{3}\right) \\
& +\left(8 H^{\prime} k P P_{3} \mu_{3} C_{56}+8 H^{\prime} k P P_{1} \mu_{1} C_{56}\right)\left(8 H^{\prime} k P^{2} C_{56} C_{66}-8 H^{\prime} k P_{1} P_{3} \mu_{1} \mu_{3} C_{56} / C_{66}\right), \\
L_{3}= & \left(8 P^{2} C_{66}{ }^{2}-8 P_{1} P_{3} \mu_{1} \mu_{3}+8 H^{\prime} k P^{2} P_{3} \mu_{3} C_{66}-8 H^{\prime} k P^{2} P_{3} C_{66}{ }^{2}+8 H^{\prime} k P^{2} P_{1} \mu_{1} C_{66}+8 H^{\prime} k P_{3}^{2} P_{1} \mu_{1} \mu_{3}\right)^{2} \\
& +\left(8 H^{\prime} k P^{2} C_{56} C_{66}-8 H^{\prime} k P_{1} P_{3} \mu_{1} \mu_{3} C_{56} / C_{66}\right)^{2} .
\end{aligned}
$$

which is the dispersion relation for $\mathrm{SH}$ waves propagating in an irregular monoclinic layer sandwiched between two isotropic half spaces. 


\section{Case 2:}

When $\mu_{1}=0$ and $m_{H}=0$ the dispersion relation (66) reduces to

$$
\tan \left\{\sqrt{\left(\frac{C^{2}}{\beta_{2}^{2}}-\frac{C_{55}}{C_{66}}+\frac{C_{56}^{2}}{C_{66}^{2}}\right)} k H\right\}=\frac{J_{4}}{L_{4}}
$$

where

$$
\begin{aligned}
J_{4}= & 2 P\left(4 H^{\prime} k P^{2} C_{66}{ }^{2}+4 H^{\prime} k P_{3}^{2} \mu_{3} C_{66}-4 P_{3} \mu_{3} C_{66}\right) \\
& \times\left(8 H^{\prime} k P^{2} P_{3} C_{66}{ }^{2}-8 P^{2} C_{66}{ }^{2}-8 H^{\prime} k P^{2} P_{3} \mu_{3} C_{66}\right)+64 H^{\prime 2} k^{2} P^{3} P_{3} \mu_{3} C_{56}{ }^{2} C_{66}, \\
L_{4}= & \left(8 H^{\prime} k P^{2} P_{3} C_{66}{ }^{2}-8 P^{2} C_{66}{ }^{2}-8 H^{\prime} k P^{2} P_{3} \mu_{3} C_{66}\right)^{2}+64 H^{\prime 2} k^{2} P^{4} C_{56}{ }^{2} C_{66}{ }^{2} .
\end{aligned}
$$

Which is the result obtained by Chattopadhyay et al. (2008) for SH waves propagating in an irregular monoclinic layer lying over an isotropic half space.

\section{Case 3:}

When $m_{H}=0$ and $H^{\prime}=0$ the dispersion relation (66) reduces to

$$
\tan \left\{\sqrt{\left(\frac{C^{2}}{\beta_{2}^{2}}-\frac{C_{55}}{C_{66}}+\frac{C_{56}{ }^{2}}{C_{66}{ }^{2}}\right)} k H\right\}=\frac{P P_{1} \mu_{1} C_{66}+P P_{3} \mu_{3} C_{66}}{P^{2} C_{66}{ }^{2}-P_{1} P_{3} \mu_{1} \mu_{3}}
$$

which is the dispersion relation for SH waves propagating in a regular monoclinic layer sandwiched between two isotropic half spaces.

\section{Case 4:}

When $C_{66}=C_{55}=\mu_{2}, C_{56}=0, m_{H}=0$ and $H^{\prime}=0$ the dispersion relation (66) reduces to

$$
\tan \left\{\sqrt{\left(\frac{c^{2}}{\beta_{2}^{2}}-1\right)} k H\right\}=\frac{P_{2} P_{1} \mu_{1} \mu_{2}+P_{2} P_{3} \mu_{3} \mu_{2}}{P_{2}^{2} \mu_{2}^{2}-P_{1} P_{3} \mu_{1} \mu_{3}}
$$

where $P_{2}=\left(\frac{c^{2}}{\beta_{2}^{2}}-1\right)^{1 / 2}$

This is the dispersion relation for SH waves propagating in a regular isotropic layer sandwiched between two isotropic half spaces.

\section{Case 5:}

When $C_{66}=C_{55}=\mu_{2}, C_{56}=0, \mu_{1}=0$ and $m_{H}=0$ the dispersion relation (66) reduces to

$$
\tan \left\{\sqrt{\left(\frac{c^{2}}{\beta_{2}^{2}}-1\right)} k H\right\}=\frac{J_{5}}{L_{5}}
$$

where

$$
\begin{aligned}
P_{2}= & \left(\frac{c^{2}}{\beta_{2}^{2}}-1\right)^{1 / 2}, \\
J_{5}= & 2 P\left(4 H^{\prime} k P_{2}^{2} \mu_{2}^{2}+4 H^{\prime} k P_{3}^{2} \mu_{3} \mu_{2}-4 P_{3} \mu_{3} \mu_{2}\right) \\
& \times\left(8 H^{\prime} k P_{2}^{2} P_{3} \mu_{2}^{2}-8 P_{2}^{2} \mu_{2}^{2}-8 H^{\prime} k P_{2}^{2} P_{3} \mu_{3} \mu_{2}\right), \\
L_{5}= & \left(8 H^{\prime} k P_{2}^{2} P_{3} \mu_{2}^{2}-8 P_{2}^{2} \mu_{2}^{2}-8 H^{\prime} k P_{2}^{2} P_{3} \mu_{3} \mu_{2}\right)^{2} .
\end{aligned}
$$

This is the result obtained by Chattopadhyay (1975) for SH waves propagating in an irregular isotropic layer lying over an isotropic half space. 


\section{Case 6:}

When $\mu_{1}=0, m_{H}=0$ and $H^{\prime}=0$ the dispersion relation (66) reduces to

$\tan \left\{\sqrt{\left(\frac{c^{2}}{\beta_{2}^{2}}-\frac{C_{55}}{C_{66}}+\frac{C_{56}{ }^{2}}{C_{66}{ }^{2}}\right)} k H\right\}=\frac{\mu_{3} P_{3}}{C_{66} P}$

which is the result obtained by Chattopadhyay and Pal (1983) for SH waves propagating in a regular monoclinic layer lying over an isotropic half space.

\section{Numerical examples}

For the case of an irregular monoclinic magnetoelastic layer between two isotropic half spaces we take the following data

(i) For upper isotropic half space, $M_{1}$ (Gubbins, 1990)

$$
\begin{aligned}
& \mu_{1}=7.10 \times 10^{10} \mathrm{~N} / \mathrm{m}^{2}, \\
& \rho_{1}=3,321 \mathrm{Kg} / \mathrm{m}^{3} .
\end{aligned}
$$

(ii) For monoclinic layer, $\mathrm{M}_{2}$ (Tiersten, 1969)

$$
\begin{array}{ll}
C_{55}=0.94 \times 10^{11} \mathrm{~N} / \mathrm{m}^{2}, & C_{56}=-0.11 \times 10^{11} \mathrm{~N} / \mathrm{m}^{2}, \\
C_{66}=0.93 \times 10^{11} \mathrm{~N} / \mathrm{m}^{2}, & \rho_{1}=7,450 \mathrm{Kg} / \mathrm{m}^{3} .
\end{array}
$$

(iii) For lower isotropic half space, $M_{3}$ (Gubbins, 1990)

$$
\begin{aligned}
& \mu_{3}=6.77 \times 10^{10} \mathrm{~N} / \mathrm{m}^{2}, \\
& \rho_{1}=3,323 \mathrm{Kg} / \mathrm{m}^{3} .
\end{aligned}
$$

Moreover, the following data are used

$$
m_{H}=\frac{\mu_{e} H_{0}^{2}}{C_{66}}=0.0,0.35,0.65,0.4,0.8 ; \phi=10^{\circ} .
$$

The effect of magnetic field and irregular boundary on the propagation of plane SH waves propagating in perfectly conducting monoclinic magnetoelastic layer with rectangular irregularity sandwiched between two isotropic elastic half spaces has been depicted by means of graphs. Figure 2 to 6 gives the variation of non-dimensional phase velocity $\left(c / \beta_{2}\right)$ with respect to nondimensional wave number $k H$ for different values of monoclinic-magnetoelastic coupling parameters $\left(m_{H}\right)$. The small change in the non-dimensional wave number produces substantial change in non-dimensional phase velocity. In each of these figures graphs are drawn for different sizes of irregularity. It is observed that as the size of irregularity $\left(H^{\prime} / H\right)$ increases, the phase velocity decreases. Also with the increase of parameter $\left(m_{H}\right)$, the phase velocity decreases. 


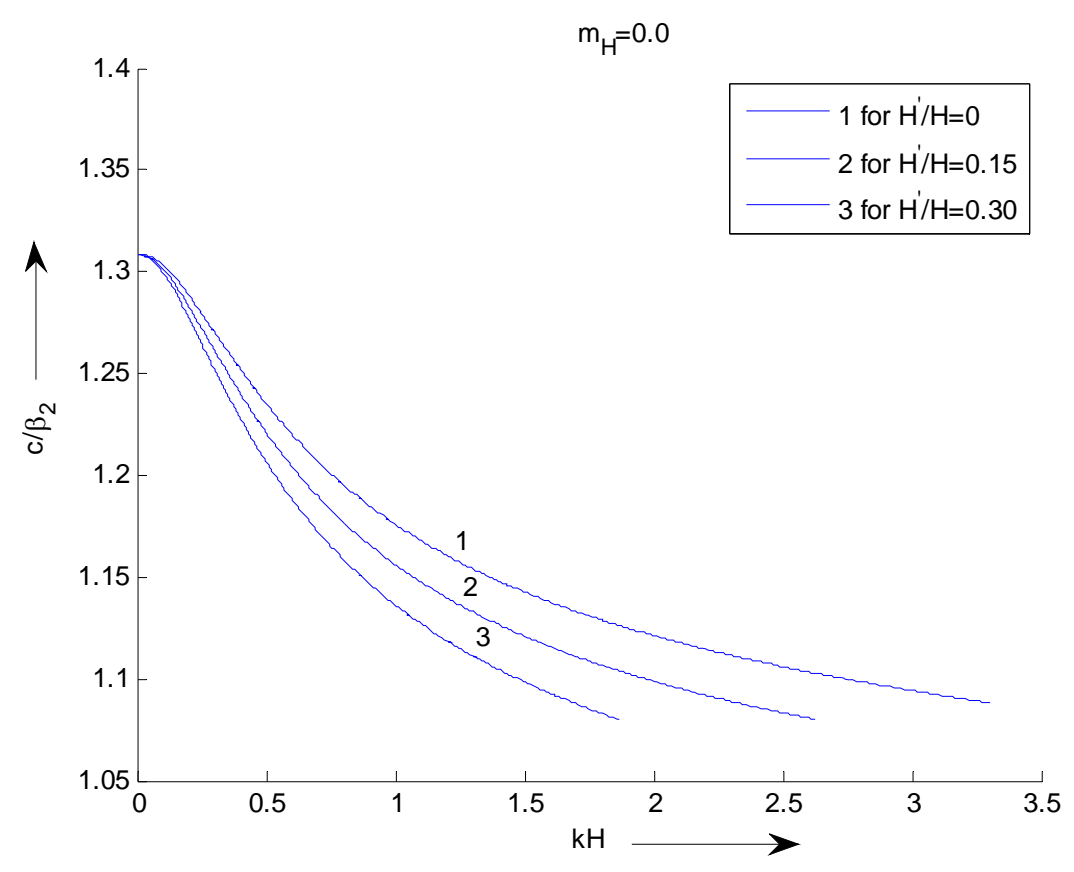

Figure 2: Dimensionless phase velocity against dimensionless wave number for $m_{H}=0.0$.

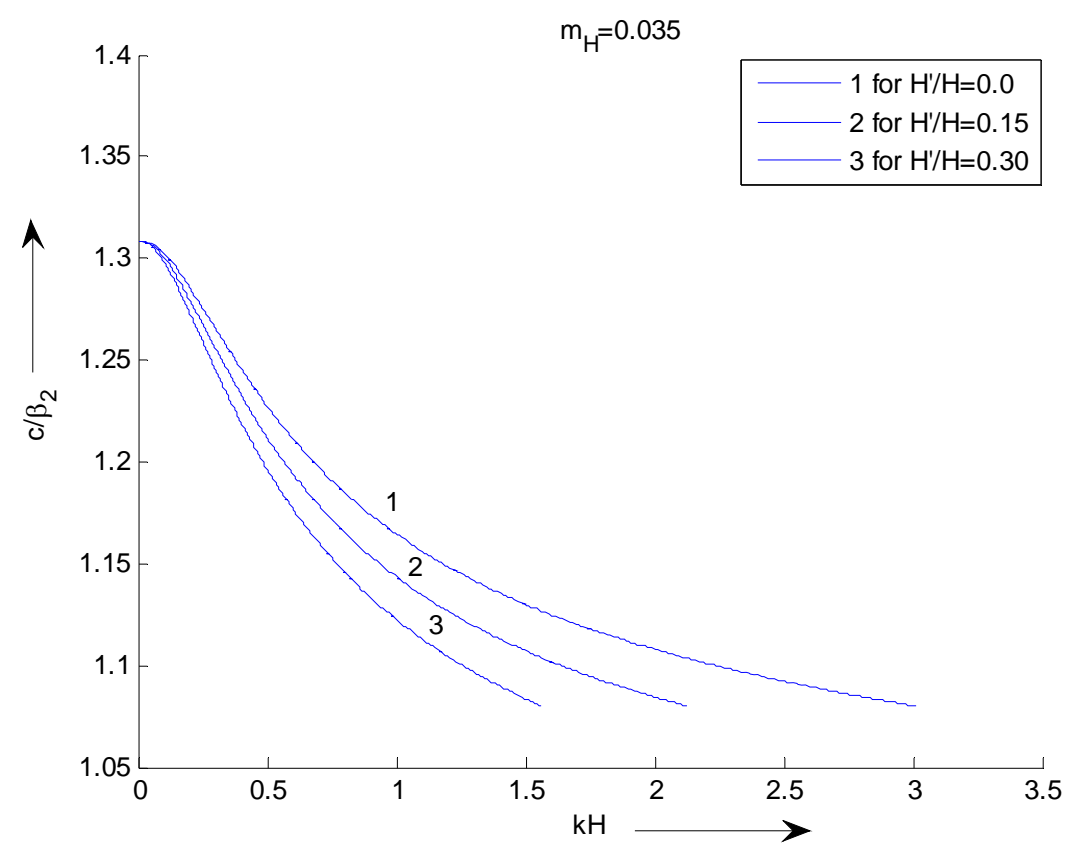

Figure 3: Dimensionless phase velocity against dimensionless wave number for $m_{H}=0.035$. 


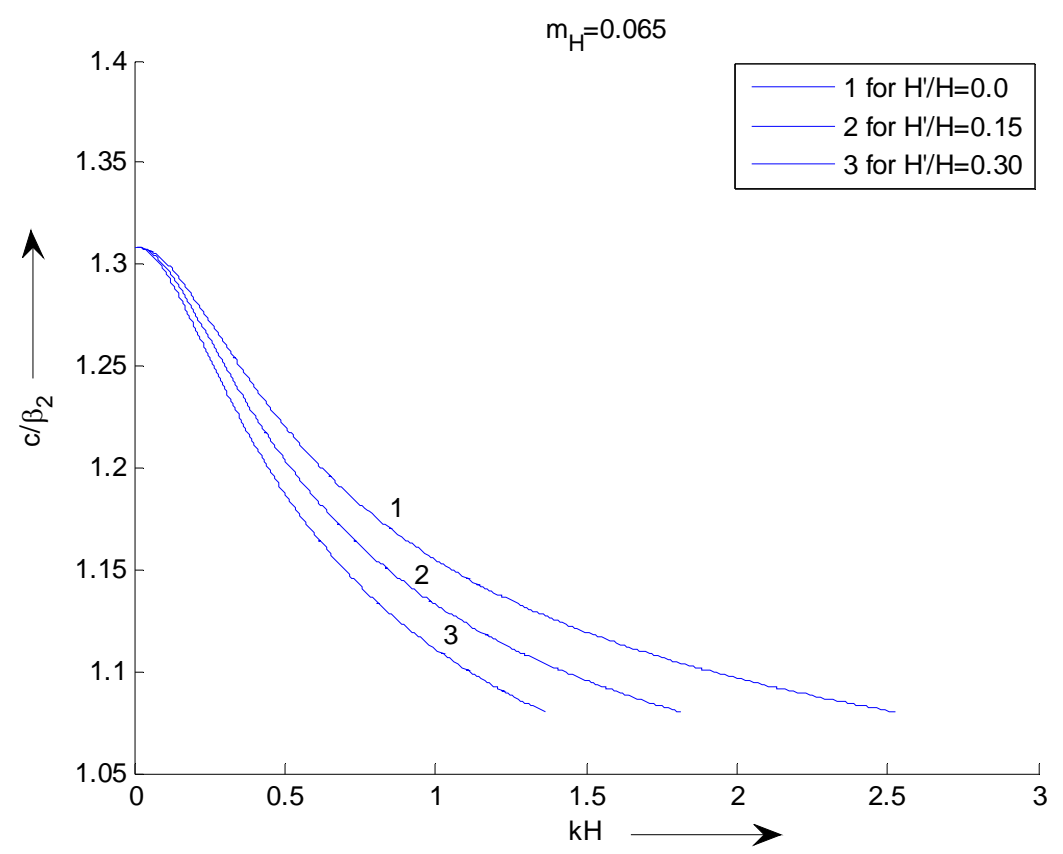

Figure 4: Dimensionless phase velocity against dimensionless wave number for $m_{H}=0.065$.

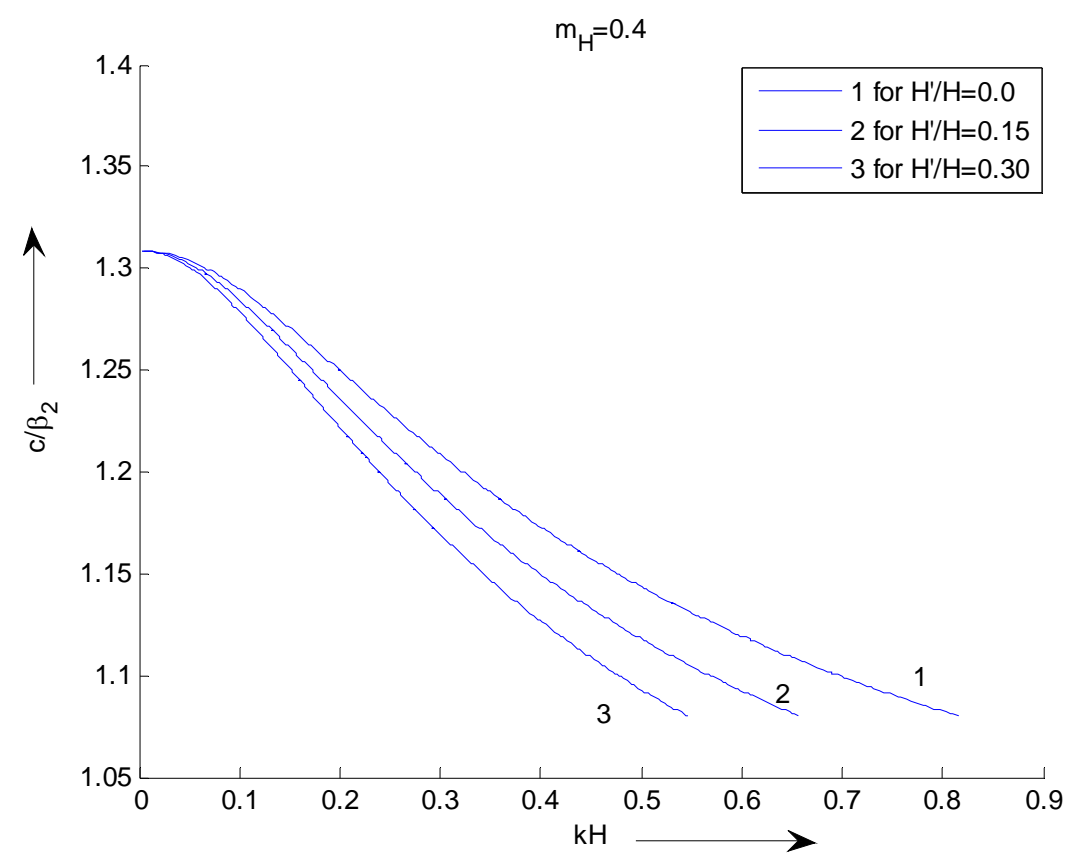

Figure 5: Dimensionless phase velocity against dimensionless wave number for $m_{H}=0.4$. 


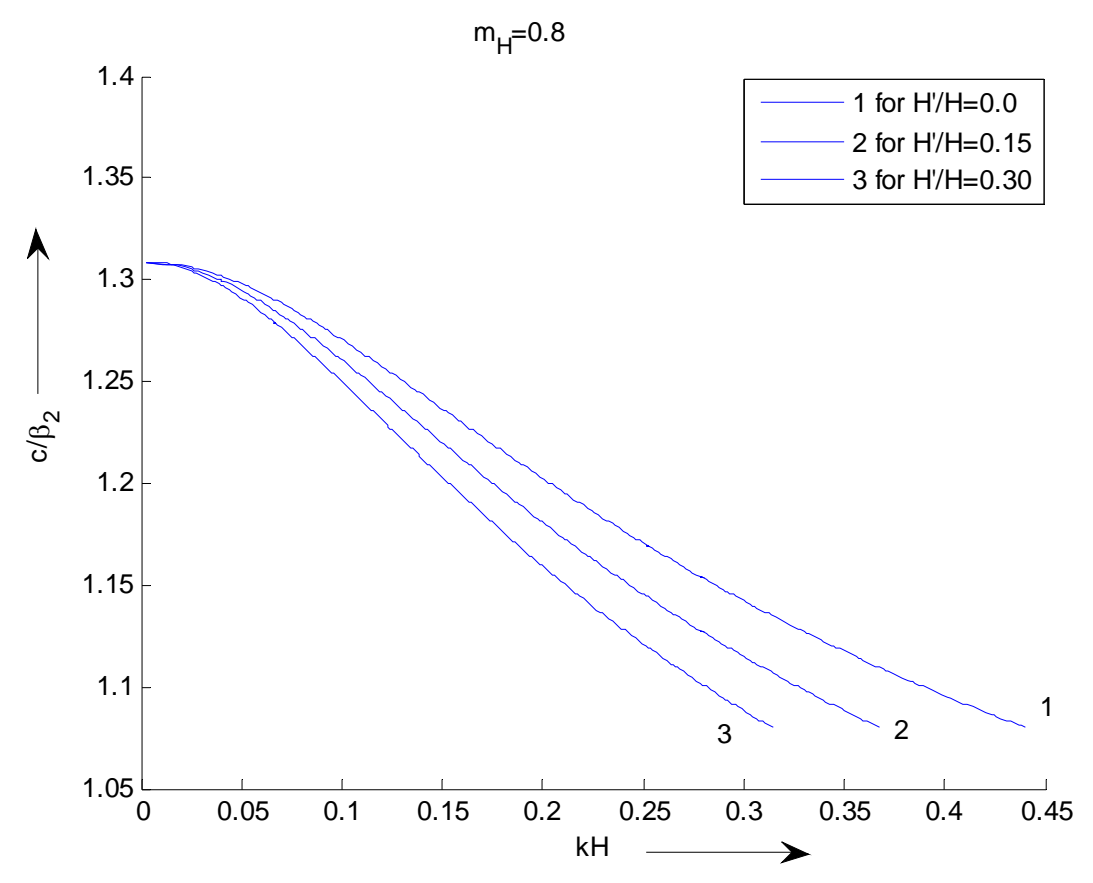

Figure 6: Dimensionless phase velocity against dimensionless wave number for $m_{H}=0.8$.

\section{Conclusion}

In this paper the dispersion relation has been obtained in the closed form. The effect of size of irregularity and magnetoelastic monoclinic parameter on the phase velocity of $\mathrm{SH}$ waves has been depicted and shown by means of graphs. From the above studies it can be concluded that in a monoclinic medium, with the increase in the size of irregularity the phase velocity decreases. Also, with the increase in the magnetoelastic monoclinic parameter the phase velocity retains the same nature. In the isotropic case, when irregularity and magnetic field are absent, the dispersion equation is matched with the classical SH wave equation. The present study has its application specially to the problem of waves and vibrations where the wave signals have to travel through different layers of different material properties and containing irregularities due to continental margin, mountain roots etc. These results can also be utilized in the interpretation and analysis of data of geophysical studies. The findings will be useful in forecasting formation details at greater depth through signal processing and seismic data analysis. The present study may be effectively utilized to generate initial data prior to exploitation of the formation. This study may be useful to geophysicist and metallurgist for analysis of rock and material structures through Non-Destructive Testing (NDT).

\section{Acknowledgement}

The authors convey their sincere thanks to Indian School of Mines University, Dhanbad for providing JRF to Mr. Abhishek Kr. Singh and also facilitating us with its best facility. Acknowledgement is also due to DST, New Delhi for the providing financial support through Project No. SR/S4/MS: 436/07, Project title: "Wave propagation in anisotropic media”.

\section{References}

Achenbach J.D., 1975. Wave propagation in elastic solids, North Holland Pub. Comp., New York.

Bhattacharya J., 1962. On the dispersion curve for Love wave due to irregularity in the thickness of the transversely isotropic crustal layer, Gerlands Beitrage zur Geophysik, Vol. 6, pp. 324-334.

Campillo M., 2006. Modeling of SH-wave propagation in an irregularly layered medium-application to seismic profiles near a dome, Geophysical prospecting, Vol. 35, No. 3, pp. 236-249.

Chattopadhyay A., 1975. On the dispersion equation for Love wave due to irregularity in the thickness of non-homogeneous crustal layer, Acta Geophysica Polonica, Vol. 23, pp. 307-317.

Chattopadhyay A., Bandyopadhyay U.K., 1986. Shear waves in an infinite monoclinic crystal plate, International Journal of Engineering Science, Vol. 24, No. 10, pp. 1587-1596.

Chattopadhyay A., Chakraborty M., Pal A.K., 1983. Effects of irregularity on the propagation of guided SH waves, Journal de Mecanique, Vol. 2, No. 2, pp. 215-225. 
Chattopadhyay A., Gupta S., Sharma V.K., Kumari Pato, 2008. Propagation of SH waves in an irregular monoclinic crustal layer, Archive of Applied Mechanics, Vol. 78, No. 12, pp. 989-999.

Chattopadhyay A., Pal A.K., 1983. Dispersion curves of SH waves caused by irregularity in the prestressed internal stratum, Acta Geophysica Polonica, Vol. 31, No. 1, pp. 37-49.

Ding G., Darvinski M., 1998. Scattering of SH-waves in multilayered media with irregular interfaces, Earthquake Engineering \& Structural Dynamics, Vol. 25, No. 12, pp. 1391-1404.

Eringen A.C., Samuels C.J., 1959. Impact and moving loads on a slightly curved elastic half space, Journal of Applied Mechanics, Vol. 26, pp. 491-498.

Ge Z., Chen X., 2007. Wave propagation in irregularly layered elastic models: a boundary element approach with a global reflection/transmission matrix propagator, Bulletin of the Seismological Society of America, Vol. 97, No. 3, pp. 1025-1031.

Ge Z., Chen X., 2008. An efficient approach for simulating wave propagation with the boundary element method in multilayered media with irregular interfaces, Bulletin of the Seismological Society of America, Vol. 98, No. 6, pp. 3007-3016.

Gubbins D., 1990. Seismology and Plate Tectonics, Cambridge University press, Cambridge.

Kalyani V.K., Sinha A., Pallavika, Chakraborty S.K., Mahanti N. C., 2008. Finite difference modelling of seismic wave propagation in monoclinic media, Acta Geophysica, Vol. 56, No. 4, pp. 1074-1089.

Kaur J., Tomar S.K., Kaushik V.P., 2005. Reflection and refraction of SH-waves at a corrugated interface between two laterally and vertically heterogeneous viscoelastic solid half-space, International Journal of Solids and Structures, Vol. 42, No. 13, pp. 3621-3643.

Singh S.S., Tomar S.K., 2007. Quasi-P waves at a corrugated interface between two dissimilar monoclinic elastic half-spaces, International Journal of Solids and Structures, Vol. 44, No. 1, pp. 197-228.

Tiersten H.F., 1969. Linear Piezoelectric plate Vibrations, Plenum Press, New York.

Tomar S.K., Kaur J., 2007. Reflection and transmission of SH-waves at a corrugated interface between anisotropic elastic and viscoelastic solid half-spaces J. Seismology, Vol. 3, pp. 235-258.

Tranter C.J., 1966. Integral Transform in Mathematical Physics, Methuen and Co. Ltd. London.

Willis H.F., 1948. A formula for expanding an integral as series, Philosophical Magazine, Vol. 39, pp. 455-459.

\section{Biographical notes}

Professor Amares Chattopadhyay is working in Indian School of Mines since 1976. He was Awarded Atomic Energy Fellowship by the Government of India during 1973-76 for Ph.D work, Post Doctoral Fellowship for research work in 'Pierre et Marie Curie University', Paris, France during 1982-1983, and INSA-Royal Society Fellowship in 1999 for research work in UK. He worked as a Visiting Professor in the TU, Vienna, Austria and also in the University of Kaiserslautern, Germany during 2002-2003. He received Michael Madhusudan Award in 2004 and Subarna and Sashibhusan Memorial Award, in 2005. Recently he became Fellow (FICDM) of a learned society.

Dr. Shishir Gupta is an Associate professor in the Department of Applied mathematics, Indian School of Mines, Dhanbad. A Gold Medalist from Ranchi University, he has had a brilliant career. He has more than 21 years of teaching experience at undergraduate and postgraduate levels in Indian School of Mines, Dhanbad. He possesses experience of guiding students of MPhil and PhD. He has published more than 45 papers in International/National journals/Proceedings. He has served as reviewer in renowned International/ National books and journals. He has also carried out several sponsored research projects.

Mr. Abhishek Kumar Singh is a Junior Research Fellow (JRF) in the Department of Applied Mathematics, Indian School of Mines (ISM), Dhanbad, Jharkhand, India. He is pursuing his PhD under the supervision of Prof. (Dr.) Amares Chattopadhyay in the field of Elastodynamics. He did his B.Sc. from University of Allahabad, Allahabad in 2005 and then switched to ISM to earn M.Sc. in Maths \& Computing (2007) and MPhil in Applied Mathematics (2008).

Mr. Sanjeev Anand Sahu is a DST-JRF (Project No. DST(39)/2008-09/223/AM) in the Department of Applied Mathematics, Indian School of Mines (ISM), Dhanbad, Jharkhand, India. He is pursuing his Ph. D. under the supervision of Prof. (Dr.) Amares Chattopadhyay in the field of Elastodynamics. He did his B.Sc. and M.Sc. from Guru Ghansidas University, Bilaspur in 2003 and 2005 respectively, and then switched to ISM for Project as well as $\mathrm{PhD}$.

Received November 2009

Accepted December 2009

Final acceptance in revised form December 2009 